\title{
Human amnion-derived mesenchymal stem cells protect against UVA irradiation-induced human dermal fibroblast senescence, in vitro
}

\author{
CHUNLI ZHANG ${ }^{*}$, HAISHEN YUCHI ${ }^{*}$, LU SUN, XIAOLI ZHOU and JINDE LIN \\ Department of Clinical Research, Friendship Plastic Surgery Hospital, \\ Nanjing Medical University, Nanjing, Jiangsu 210029, P.R. China
}

Received November 11, 2016; Accepted June 6, 2017

DOI: $10.3892 / \mathrm{mmr} .2017 .6795$

\begin{abstract}
The aim of the present study was to determine if human amnion-derived mesenchymal stem cells (HAMSCs) exert a protective effect on ultraviolet A (UVA) irradiation-induced human dermal fibroblast (HDF) senescence. A senescence model was constructed as follows: HDFs $\left(10^{4}-10^{6}\right.$ cells/well) were cultured in a six-well plate in vitro and then exposed to UVA irradiation at $9 \mathrm{~J} / \mathrm{cm}^{2}$ for $30 \mathrm{~min}$. Following the irradiation period, HDFs were co-cultured with HAMSCs, which were seeded on transwells. A total of $72 \mathrm{~h}$ following the co-culturing, senescence-associated $\beta$-galactosidase staining was performed and reactive oxygen species (ROS) content and mitochondrial membrane potential $(\Delta \psi \mathrm{m})$ were detected in the HDFs via flow cytometric analysis. The results demonstrated that the percentage of HDFs, detected via staining with X-gal, were markedly decreased when co-cultured with human HAMSCs, compared with the group that were not co-cultured. The ROS content was decreased and the mitochondrial membrane potential $(\Delta \psi \mathrm{m})$ recovered in cells treated with UVA and HAMSCs, compared with that of cells treated with UVA alone. Reverse transcription-quantitative polymerase chain reaction revealed the significant effects of HAMSCs on the HDF senescence marker genes p53 and matrix metalloproteinase-1 mRNA expression. In addition to this, western blot analysis verified the effects of HAMSCs on UVA induced senescence, providing a foundation for novel regenerative therapeutic methods. Furthermore, the results suggested
\end{abstract}

Correspondence to: Professor Jinde Lin, Department of Clinical Research, Friendship Plastic Surgery Hospital, Nanjing Medical University, 146 Hanzhong Road, Nanjing, Jiangsu 210029, P.R. China E-mail: hzljd@sohu.com

*Contributed equally

Key words: human amnion-derived mesenchymal stem cells, human dermal fibroblasts, UVA irradiation senescence that activation of the extracellular-signal regulated kinase $1 / 2$ mitogen activated protein kinase signal transduction pathway, is essential for the HAMSC-mediated UVA protective effects. The decrease in ROS content additionally indicated that HAMSCs may exhibit the potential to treat oxidative stress-mediated UVA skin senescence in the future.

\section{Introduction}

Skin is an essential natural barrier protecting body from physical, chemical and microbial hazards, also a visual indicator of body's aging process (1). 90\% of the ultraviolet (UV) reaching surface of the earth is long-wavelength irradiation (UVA, 320-400 nm), which can penetrate the epidermis into the dermis. So it is well established that UVA is responsible for skin photoaging induced by UV. Studies demonstrated UVA radiation can induce cytokine expression in human epidermoid carcinoma cells. UVA radiation caused an increased proportion of $\beta$-Gal positive cells and reduced telomere length in human skin fibroblasts. In addition, UVA radiation inhibited TGF- $\beta 1$ secretion, induced G1 phase arrest, reduced SOD and GSH-Px levels, increased MDA levels and the expression of MMP-1, TIMP-1, p66, p53 mRNA expression (2,3).

Human amnion-derived mesenchymal stem cells (HAMSCs) obtained from human amniotic membrane (AM) are readily available and high abundant tissue, with substantial benefits as seed cells $(3,4)$. Their low anti-flammatory properties and fewer ethical concerns compared with other sources of stem cell are clear advantage (5-7). It is proved that HAMSCs secrete a variety of cytokines, which is essential to a series of basic biological processes of cells (8). Recent studies revealed that HAMSCs have important roles in cell differentiation (9-12), promoting cell proliferation (13-15), enhancing cell viability and function (16-18), protecting cells from adverse effects and inhibiting apoptosis $(19,20)$ in vivo or in vitro. The present study aimed to determine whether HAMSCs involved in the protection of human dermal fibroblasts (HDFs) from UVA-induced senescence.

In order to investigate the protective mechanisms of HAMSCs against UVA-induced HDFs senescence, an in vitro cell-senescence model was built through the exposure of pre-HAMSCs-treated HDFs to UVA, and the effects of 
HAMSCs on ROS contents and mitochondrial membrane potential $(\Delta \psi \mathrm{m})$, HDFs senescence marker genes p53 and MMP1 (21) expression were detected by reverse transcription quantitative polymerase chain reaction analysis and western blot. Furthermore, senescence-associated $\beta$-galactosidase (SA- $\beta-$ Gal) staining was performed to evaluate the senescence status of HDFs. SA- $\beta$-Gal activity distinguishes senescent cells from those terminally differentiated, therefore act as a senescence biomarker. Our results showed that HAMSCs up-regulated MERK1/2 in UVA induced senescence HDFs, which means skin senescence might related to ERK1/2 MAPK signal pathway.

\section{Materials and methods}

Chemicals and reagents. Fetal bovine serum (FBS), $\alpha$-minimum essential medium ( $\alpha \mathrm{MEM})$, trypsin-EDTA, phosphate-buffered saline (PBS) and penicillin G-streptomycin sulfate were purchased from Gibco Life Technologies (Carlsbad, CA, USA). 2,7-dichlorodihydro fluoresceindiacetate (DCFH-DA) fom Sigma-Aldrich (St. Louis, MO, USA). Transwells (6-well millicell Hanging Cell Culture Inserts, $0.4 \mu \mathrm{m}, \mathrm{PET}$ ) and 6-well culture plates were purchased from Millipore Corp. (Billerica, MA, USA). The goat anti rabbit IgG, phosphor-p44/42 MAPK rabbit mAb (p-ERK1/2), JNK MAPK rabbit $\mathrm{mAb}$, 53 rabbit $\mathrm{mAb}$, SIRT1 rabbit $\mathrm{mAb}$ and Senescence $\beta$-Galactosidase Staining kit were purchased from Cell Signaling Technology, Inc. (3 Trask Lane; Danvers, MA, USA). Penicillin and streptomycin from Gibco Life Technologies. Other reagents used were of the highest commercial grade available.

Cell culture. Human amnion-derived mesenchymal stem cells were prepared as described previously (3). Briefly, human amniotic membrane was mechanically peeled off from the chorion of a placenta obtained from an uncomplicated elective caesarean section with the informed consent of the donor patient. The HAMSCs layer was thoroughly scraped out from the underlying tissues such as the spongy and fibroblast layers. Within $24 \mathrm{~h}$ AM layer was then treated with $0.125 \%$ trypsin three times each for $20 \mathrm{~min}$ to obtain dissociated HAMSCs. The cells were cultured in $\alpha$-MEM supplemented with $10 \% \mathrm{FBS}$, penicillin $(100 \mathrm{U} / \mathrm{ml})$ and streptomycin $(100 \mu \mathrm{g} / \mathrm{ml})$, incubated in an incubator at $37^{\circ} \mathrm{C}$ with $5 \% \mathrm{CO}_{2}$ in a humidified atmosphere. The culture medium was changed every 3 days.

Primary HDFs were purchased from Wuxi BioHermes Bio\&Medical Technology Co., Ltd. (Wuxi, China). Cultured in a $10-\mathrm{cm}$ dish in $\alpha$-MEM supplemented with $10 \%$ fetal bovine serum, penicillin $(100 \mathrm{U} / \mathrm{ml})$ and streptomycin $(100 \mu \mathrm{g} / \mathrm{ml})$.

The Co-culture system. The transwell co-culture system was used to investigate the effects of HAMSCs on HDFs. HDFs were seeded at an initial density of $5 \times 10^{4}$ cells $/ \mathrm{cm}^{2}$ in 6 -well culture plates. Transwells were placed in other 6-well culture plates and seed at increasing HAMSCs $\left(5 \times 10^{4}\right.$ cells/transwell, $10 \times 10^{4}$ cells/transwell and $15 \times 10^{4}$ cells/transwell). Immediately after $9 \mathrm{~J} / \mathrm{cm}^{2} \mathrm{UVA}$ on HDFs to create UVA induced senescence, HAMSCs in transwells moved into the appropriate well of 6-well plate to co-culturing with HDFs.
HDFs in wells with HAMSCs on transwells served as the treatment groups, while HDFs without transwells were designated as the control group.

UVA irradiation. $24 \mathrm{~h}$ after HDFs seeded in the six-well plate, HDFs were exposed to $9 \mathrm{~J} / \mathrm{cm}^{2}$ (30 min) UVA irradiation. Cells were washed with phosphate-buffered saline (PBS) and covered with a thin layer of PBS prior to UVA exposure. The culture plate lid was removed, and the 6-well plate was placed on a brass block embedded on ice, in order to reduce any evaporation, at a distance of $15 \mathrm{~cm}$ from the UVA light source. As the UVA irradiation source, an Ultraviolet phototherapy instrument (SS-04A; Shanghai Sigma High-tech Co., Ltd., Shanghai, China) equipped with a $15-\mathrm{W}$ ozone-free UVA lamp (CEL015 W; Philips, Groningen, The Netherlands) was used. The incidence dose of UVA was measured with a UVA/UVB-ultraviolet meter (Factory affiliated to Beijing Normal University, Beijing, China). After UVA irradiation, PBS was replaced with culture medium and transwells seeded with HAMSCs were placed in wells of the co-culture group, then they were incubated under standard conditions for $72 \mathrm{~h}$ prior to analysis.

Analysis of cellular proliferation. HDFs accepted UVA irradiation then co-cultured with HAMSCs after $72 \mathrm{~h}$, transwells containg HAMSCs were removed and HDFs were harvested. After fixed with $75 \%$ ice-cold ethanol at $4^{\circ} \mathrm{C}$ in the dark, cell cycle fractions (G0, G1, and G2, M phase) were determined by flow cytometry.

SA- $\beta$-Gal staining. SA- $\beta$-Gal activity was evaluated using a $\beta$-galactosidase staining kit (Beyotime Institute of Biotechnology, Haimen, China). Cells were washed with PBS and fixed for $15 \mathrm{~min}$ at room temperature with fixative solution. The HDFs cells were then incubated at $37^{\circ} \mathrm{C}$ overnight. SA- $\beta$-Gal-positive staining was expressed as a percentage of the total number of cells; cell numbers were counted in four continuous visual fields using a microscope (Olympus CX51; Olympus, Tokyo, Japan; total magnification, x20).

Assessment of ROS production. The level of ROS induced by UVA in HDFS was measured using DCFH-DA as a fluorescent probe. After irradiation and co-culturing with HAMSCs, transwells were removed and HDFs were washed three times with PBS, incubated with DCFH-DA $(10 \mathrm{mM})$ for $30 \mathrm{~min}$ at $37^{\circ} \mathrm{C}$, washed three times with PBS. Macrographs of DCFDA fluorescence were immediately.

Flow cytometry analysis of mitochondrial membrane potential. Mitochondrial membrane potential $(\Delta \psi \mathrm{m})$ was analyzed by a fluorescent dye JC-1 (Beyotime Institute of Biotechnology), following manufactur's protocol. JC-1 is capable of selectively entering mitochondria where it forms monomers and emits green fluorescence $(530 \mathrm{~nm})$ when $\Delta \psi \mathrm{m}$ is relatively low. At high $\Delta \psi \mathrm{m}, \mathrm{JC}-1$ aggregates and gives a red fluorescence $(590 \mathrm{~nm})$. Assays were initiated by incubating HDFs with $\mathrm{JC}-1$ for $30 \mathrm{~min}$ at $37^{\circ} \mathrm{C}$ in the dark and the fluorescence of separated cells was detected with a flow cytometer (FAC-SCalibur; BD Biosciences, San Diego, CA, USA). $\Delta \psi \mathrm{m}$ was determined by a ratio of fluorescence intensity at $590 \mathrm{~nm}$ 

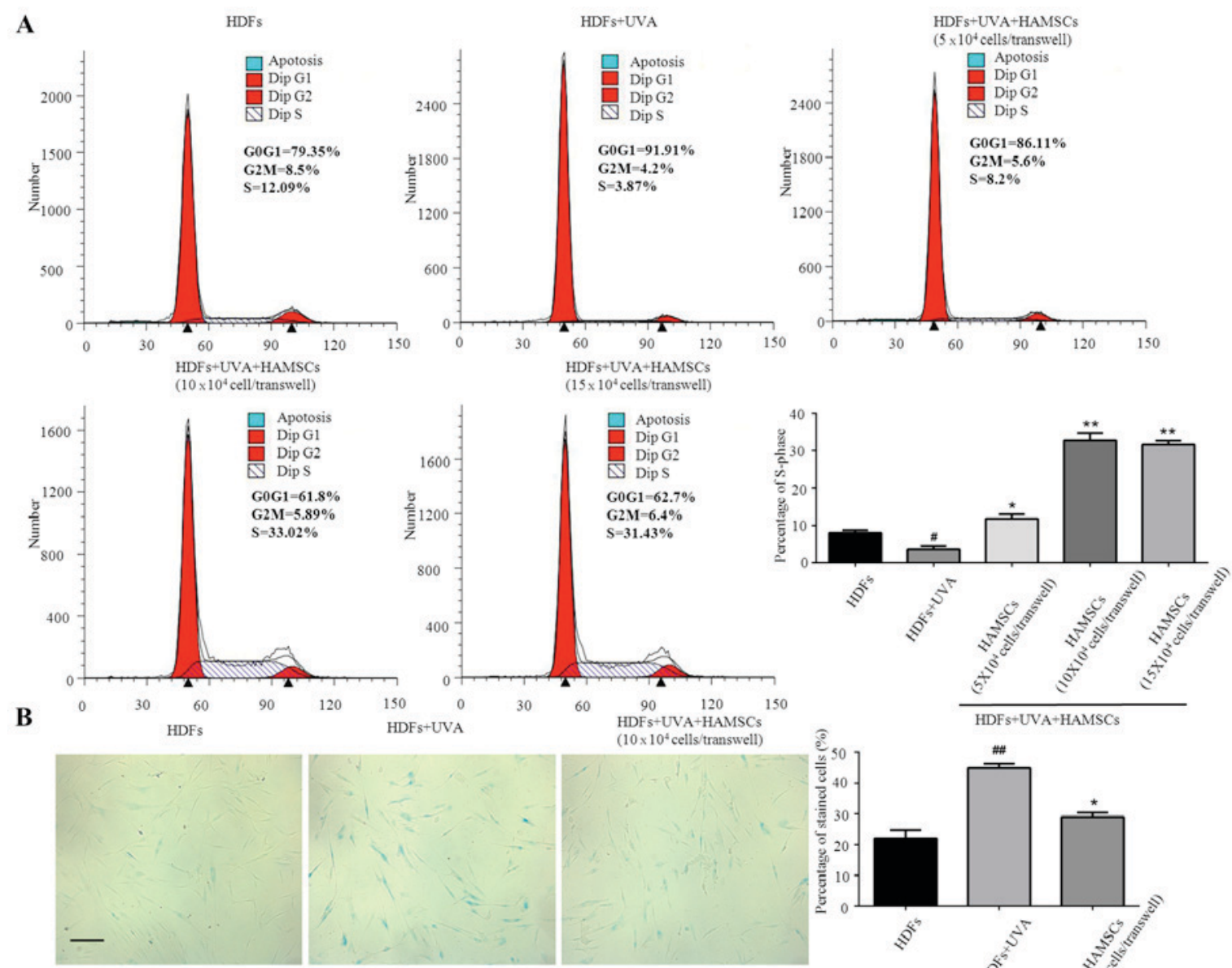
$\left(10 \times 10^{4}\right.$ cells stranswell)

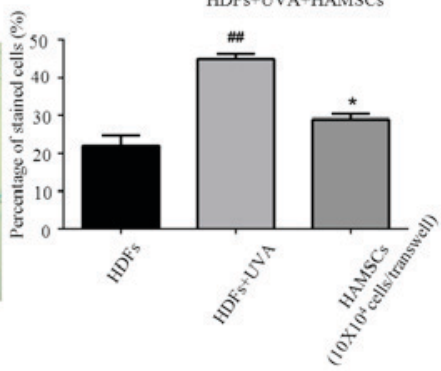

Figure 1. The effect of HAMSCs on UVA induced HDFs proliferation and senescence by flow cytometry and X-gel staining. Transwells containing HAMSCs were moved into the correlating wells containing HDFs which accepted $9 \mathrm{~J} / \mathrm{cm}^{2} \mathrm{UVA}$ irradiation. (A) HDFs cell cycle fractions (G0, G1, S, and G2, M phase) were detected at $72 \mathrm{~h}$ after UVA irradiation and co-culture or without HAMSCs. (B) SA- $\beta$-gal staining was taken $72 \mathrm{~h}$ after irradiation and co-culturing with/without HAMSCs, the stained surface was measured by image-pro plus 6.0 (IPP) analysis. Values are means \pm SD of 3 independent experiments with each performed in triplicate. ${ }^{\#} \mathrm{P}<0.05$ vs. the HDFs group; ${ }^{\# \prime} \mathrm{P}<0.01$ vs the HDFs group; ${ }^{*} \mathrm{P}<0.05$ and ${ }^{* *} \mathrm{P}<0.01$ compared with the group treated with UVA alone.

to that at $530 \mathrm{~nm}$. A minimum of 10,000 cells per sample was acquired and analyzed.

Assessment of senescence related RNA and protein. Total RNA was extracted from the cells using TRIzol reagent (Promega Corp., Madison, WI, USA). RNA concentration and purity were determined with a Nanodrop 2000-UV spectrophotometer (Thermo Fisher Scientific, Inc., Waltham, MA, USA). Ribosomal RNA band integrity was evaluated using conventional denaturing agarose gel electrophoresis using the SDS-PAGE gel quick preparation kit (Beyotime Institute of Biotechnology). Equal amounts of RNA (500 ng) from each sample were reverse transcribed using a PrimeScript ${ }^{\mathrm{TM}}$ RT Reagent kit with gDNA Eraser (Takara Bio, Dalian, China) according to the manufacturer's instructions. qPCR was performed using SYBR-Green dye method (Premix Ex Taq; Takara Bio) using an ABI700 Real-Time PCR detection system (Applied Biosystems; Life Technologies, Thermo Fisher Scientific, Inc.). The following standard cycling conditions for qPCR were applied: $95^{\circ} \mathrm{C}$ for $3 \mathrm{~min}$ to activate polymerase, 40 cycles of denaturation at $95^{\circ} \mathrm{C}$ for $15 \mathrm{sec}$ and annealing-extension at $60^{\circ} \mathrm{C}$ for $30 \mathrm{sec}$. Melting curve analysis was performed following every run by defined heating up to $95^{\circ} \mathrm{C}$ to assess the presence of unspecific PCR products. Specific primers for the RT-qPCR reactions were as follows: MMP1 forward, 5'-TTGGAGGGGATGCTCATT-3' and, reverse, 5'-TAAAACGCAGCTCAGTAACAGTCCG-3'; p53, forward, 5'-AGAATCTCCGCAAGAAAGG-3', reverse, 5'-GCTGGTATGTCCTACTCCC-3'; $\beta$-actin, forward, 5'-TGG AATCTTGCTCTTATTTTCACA-3' and reverse, 5'-TAA AACGCAGCTCAGTAACAGTCCG-3'. All primers were synthesized by Sangon Biotech, Co., Ltd. (Shanghai, China) and used at $400 \mathrm{nM}$ expect for $\beta$-actin at $300 \mathrm{nM}$. All PCR efficiencies were between 90 and $110 \%$.

At the end of $72 \mathrm{~h}$ after UVA irradiation and co-culture, transwells containg HAMSCs were removed and HDFs in each group were lysed in RIPA buffer containg $1 \mathrm{mM}$ phenylmethane sulfonylfuoride according to the manufacturer's instructions. The total protein concentration was determined using a bicinchoninic acid (BCA) assay kit. Protein lysates $(20 \mu \mathrm{g})$ were separated by sodium dodecyl sulfate-polyacrylamide gel electrophoresis (SDS-PAGE) and then transferred onto $0.22 \mu \mathrm{m}$ polyvinylidene difluofide membranes (Millipore Corp.). After blocking, membranes were incubated overnight at $4^{\circ} \mathrm{C}$ with specific antibodies for the detection of p53 (1:1,000), p38 (1:1,000), SIRT1 (1:1,000), p-ERK1/2 (1:500), ERK1/2 

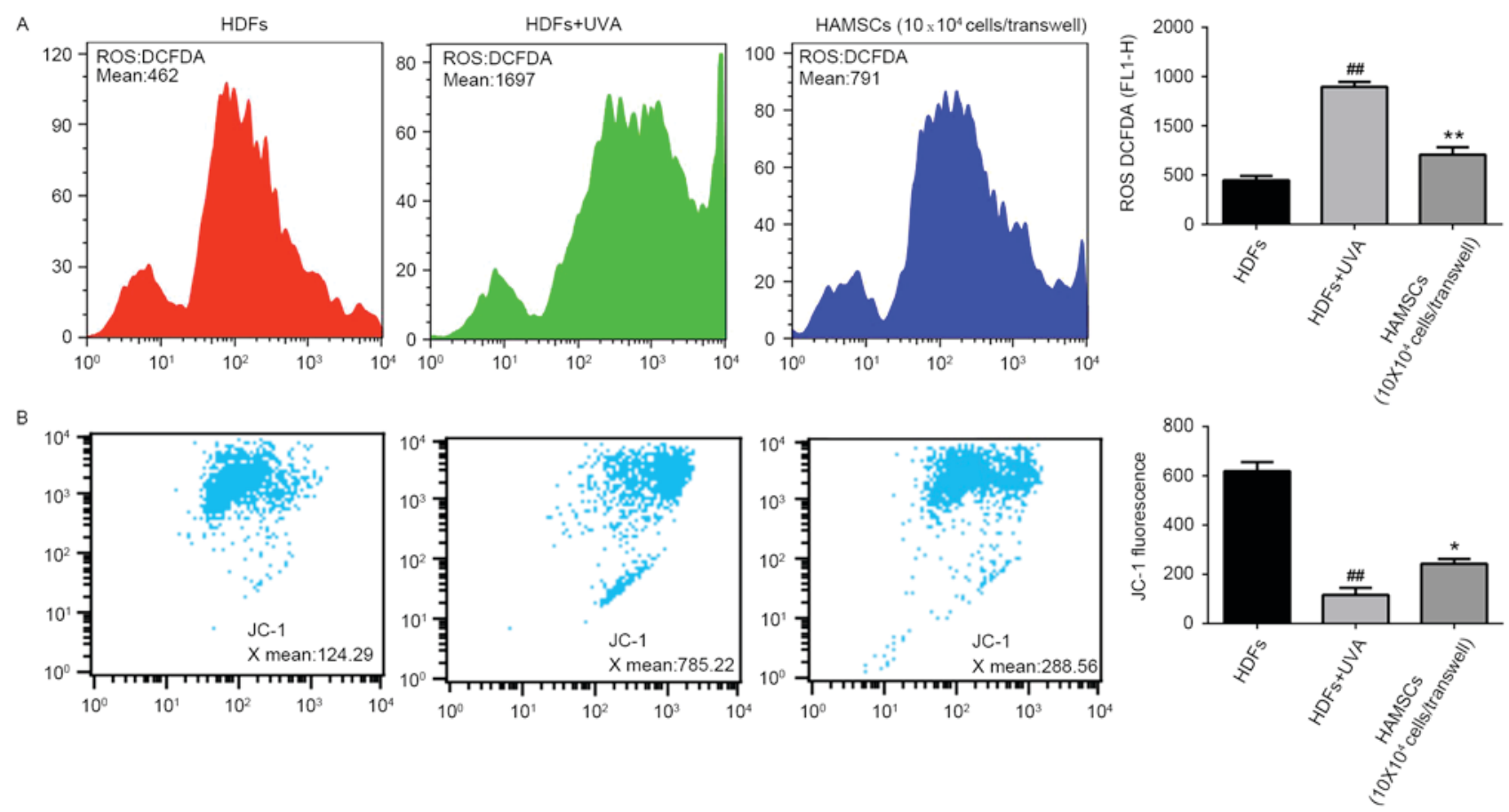

Figure 2. Flow cytometry of ROS generation and mitochondria depolarization in UVA irradiated HDFs after co-culturing with or without HAMSCs for $72 \mathrm{~h}$ or without. Transwells containing HAMSCs were moved into the correlating wells containing HDFs which just accepted $9 \mathrm{~J} / \mathrm{cm}^{2} \mathrm{UVA}$ irradiation. (A) Macrogaphs of DCFDA fluorescence was determined by flow cytometry at $72 \mathrm{~h}$ after UVA irradiation and co-culturing with/without HAMSCs. (B) JC-1 fluorescence was determined by flow cytometry at $72 \mathrm{~h}$ after UVA irradiation and co-culture with or without HAMSCs. ${ }^{\# \#} \mathrm{P}<0.01 \mathrm{vs}$ the HDFs group; ${ }^{*} \mathrm{P}<0.05$ and ${ }^{* *} \mathrm{P}<0.01$ compared with the group treated with UVA alone. Values are means \pm SD of 3 independent experiments with each performed in triplicate.

(1:500). After three washes with PBST (0.5\% Tween-20 in PBS), the membranes were incubated with the relevant secondary antibodies $(1: 2,000)$ for $1 \mathrm{~h}$ at $37^{\circ} \mathrm{C}$, washed and visualized with an ECL detection kit (Amersham Pharmacia Biotech, Piscataway, NJ, USA). The GAPDH (1:500) served as internal control.

Statistical analysis. Analyses were performed using GraphPad Prism software (GraphPad Inc., La Jolla, CA, USA). Values are presented as the mean \pm standard deviation. The one-way analysis of variance was used for comparisons involving more than two groups. $\mathrm{P}<0.05$ was considered to indicate a statistically significant difference.

\section{Results}

HAMSCs promoted UVA induced HDFs proliferation and reduce UVA induced HDFs senescence. Flow cytometry were used to measure the proliferation of UVA treated HDFs seeded in the 6-well plates co-culture with HAMSCs. Cell cycle fractions (G0, G1, S, and G2, M phase) were determined by flow cytometry at $72 \mathrm{~h}$ after UVA and HAMSCs treatment. The $\mathrm{S}$ phase showed significant inhibited treated by UVA, after co-culture with HAMSCs the S-phase checkpoints increased (Fig. 1A). So we chose $10 \times 10^{4}$ cells/transwell HAMSCs in following experiment. Our results further demonstrated that co-culturing with HAMSCs accelerated UVA-induced HDFs proliferation.

$\mathrm{X}$-gal staining results showed that the percentage of cells stained by X-gal following $9 \mathrm{~J} / \mathrm{cm}^{2}$ UVA irradiation was markedly increased compared with that of the control group (10.8 and 22.6\%, respectively; $\mathrm{P}<0.05$ ), while HAMSCs attenuated the ratio of positive staining compared with that of the UVA-treated only cells (15.3 and $22.6 \%$, respectively; $\mathrm{P}<0.05$ ) (Fig. 2B).

HAMSCs inhibited ROS generation and mitochondria depolarization in UVA induced HDFs. To elucidate whether the beneficial effects of HAMSCs were linked to their antioxidant properties, the ROS generation in UVA-induced HDFs were measured. Subsequently, the intensity of fluorescence was determined by flow cytometry.

As shown in Fig. 2A, after UVA irradiation, intracellular ROS generation increased significantly. The level of ROS in UVA treated cells was much higher than the level of ROS in control cells throughout the experiment. Co-culture with HAMSCs significantly inhibited the elevated intracellular concentration of ROS.

Loss of mitochondrial membrane potential in cells has been estimated using JC-1 assay kit. In normal cells, JC-1 aggregated in mitochondria and the ratio was 124.29. UVA irradiation treated cells showed the higher ratio 785.22, which indicated the dissipation of $\Delta \psi \mathrm{m}$. HDFs treatedwith UVA and co-cultured with HAMSCs demonstrated attenuation of the dissipation of $\Delta \psi \mathrm{m} 288.56$ (Fig. 2B). Above results uggested that HAMSCs protect mitochondria depolarization induced by UVA irradiation.

HAMSCs reduced the expression of senescence related markers. Reverse transcription quantitative polymerase chain reaction analysis showed mRNA expression levels of p53 and MMP1 were significantly reduced in UVA-treated HDFs co-cultured with HAMSCs than that of the UVA-treated 

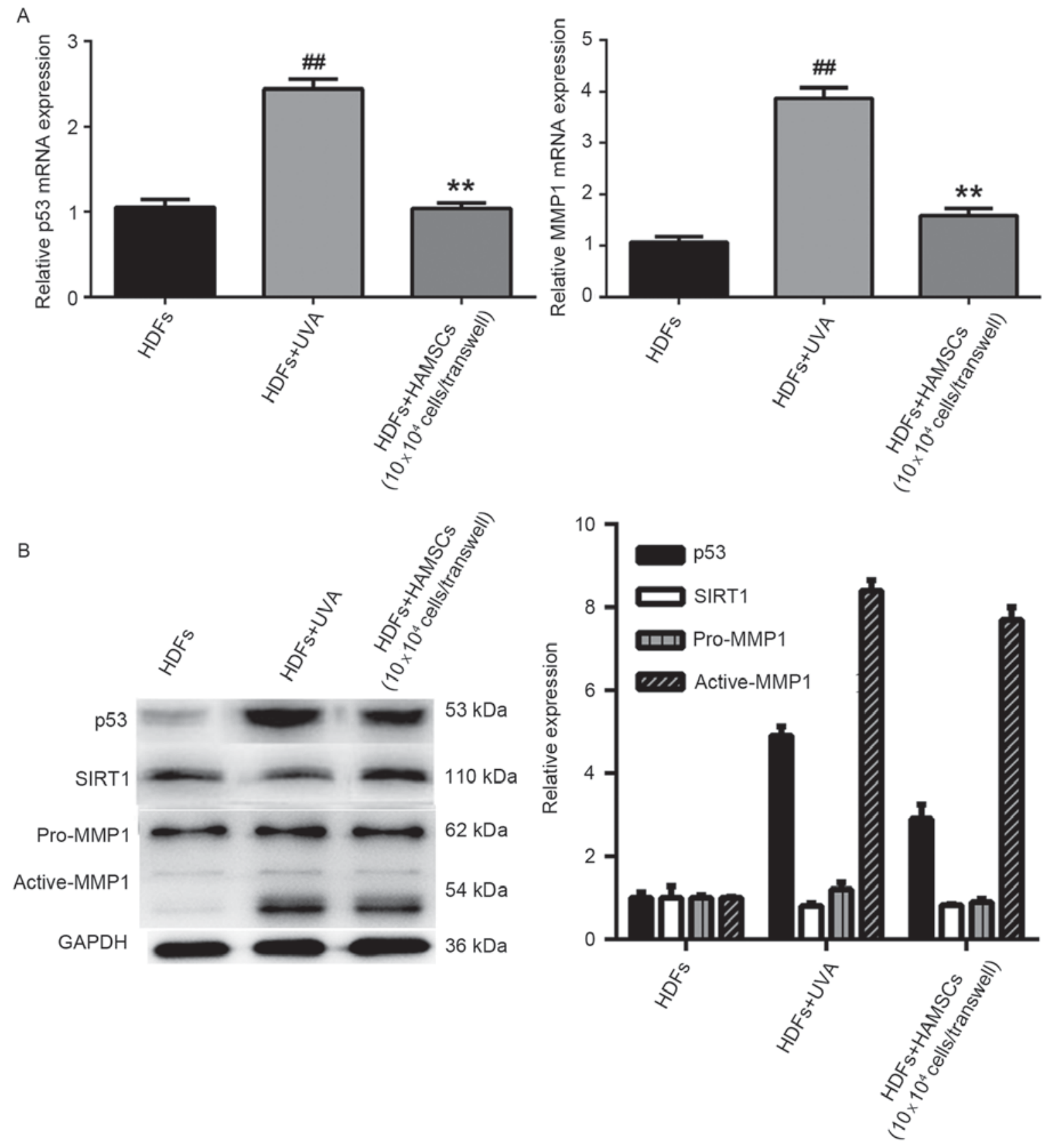

Figure 3. Expression of senescence related markers. Transwells containing HAMSCs were moved into the correlating wells containing HDFs which accepted $9 \mathrm{~J} / \mathrm{cm}^{2}$ UVA irradiation. (A) mRNA expression of MMP1 and p53 in UVA irradiated HDFs co-culturing with or without HAMSCs for $72 \mathrm{~h}$. (B) Expression of p53, MMP1 and SIRT1 in UVA irradiated HDFs co-culturing with or without HAMSCs were determined by western blot, GAPDH served as an internal control. Values are means \pm SD of 3 independent experiments with each performed in triplicate. ${ }^{\# \#} \mathrm{P}<0.01$ vs the HDFs group; ${ }^{* *} \mathrm{P}<0.01$ compared with the group treated with UVA alone.

only group $(\mathrm{P}<0.05)$ (Fig. 3A). In order to further study the efficacy of HAMSCs, western blot analysis was to evaluate the protein expression in co-culture with UVA and HAMSCs or with UVA only of HDFs. The result showed that HAMSCs had a significant effect on p53, active-MMP1 and SIRT1 (Fig. 2B).

HAMSCs activated ERK1/2 in UVA induced HDFs. EEK1/2 are important members of the MAPK signal pathway, which regulates the differentiation, mineralization and proliferation of HDFs. Fig. 4 showed ERK1/2 in HDFs in respective of UVA treatment after $72 \mathrm{~h}$ with or without co-culture with HAMSCs. Higher level of phosohorylated ERK1/2 were observed compared with UVA irradiated HDFs co-cultured with HAMSCs than without. These results suggested that HAMSCs enhance UVA-induced inhibition of ERK1/2, which might play a role in regulating UVA induced HDF senescence.

\section{Discussion}

HAMSCs has been the shining star in cell-based therapy in recent years, which appear to have several advantages over other stem cell lineages as a cell therapy. Studies showed that HAMSCs can maintain mouse spermatogonial stem cells in an undifferentiated state when cultured long term 


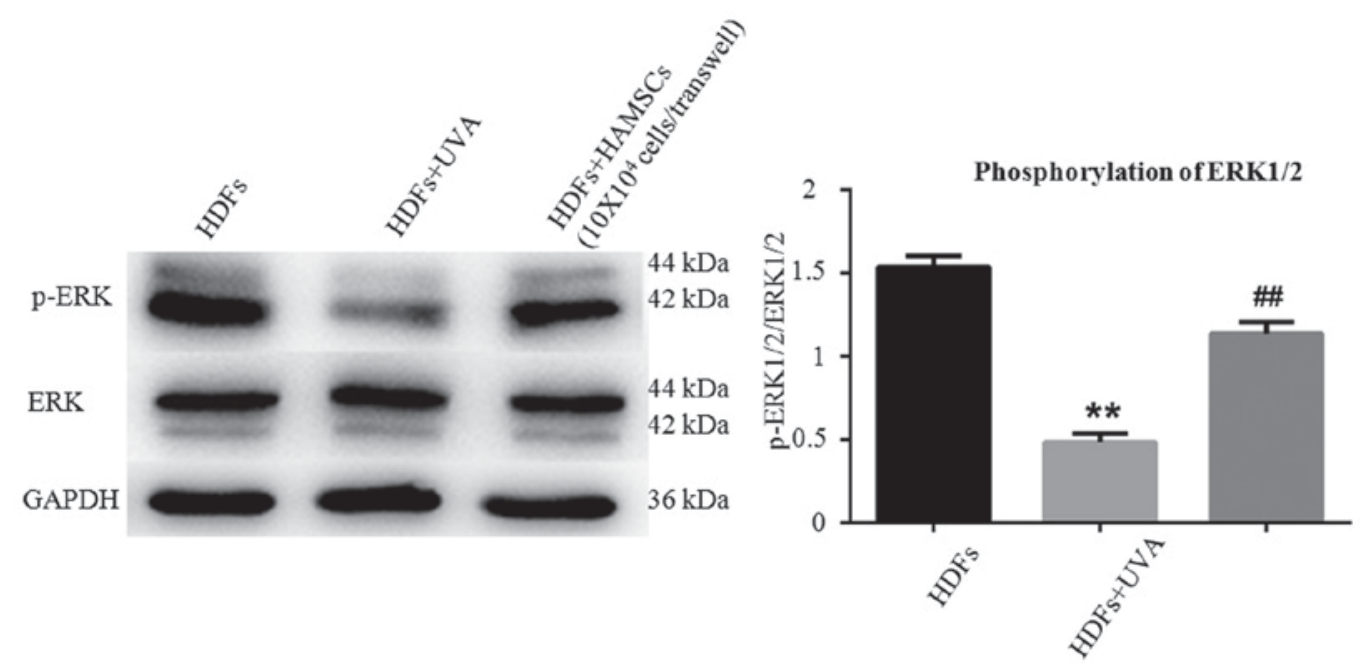

Figure 4. Expression of ERK1/2, p-ERK1/2 in UVA irradiated HDFs after co-culturing with or without HAMSCs for $72 \mathrm{~h}$. Transwells containing HAMSCs were moved into the correlating wells containing HDFs which accepted $9 \mathrm{~J} / \mathrm{cm}^{2} \mathrm{UVA}$ irradiation. Densitometric measures of the band intensity are expressed as the signal ratios indicating the level of ERK phosphorylation (p-ERK1/2 ERK1/2). ${ }^{\# \#} \mathrm{P}<0.01$ vs the HDFs group; ${ }^{* *} \mathrm{P}<0.01$ compared with the group treated with UVA alone. Values are means \pm SD of 3 independent experiments with each performed in triplicate.

due to high leukemia inhibitor factor (LIF) expression. HAMSCs had an increased proliferative capacity, higher colony-forming efficiency, fewer apoptotic cells, and similar cell-junction formation capabilities and pump functionality compared with primary HCECs $(21,22)$. Amniotic membrane can restrict dedifferentiation of human retinal pigment epithelial cells (RPE cells) in culture, promoting RPE65, CRALBP, VEGF, CD68, and tyrosinase gene expression in RPE cells (23). Experiments have demonstrated the ability of HAMSCs to migrate into brain, prevents the degeneration of nigral dopmineneurons in rats with 6-hydroxydopami-ne lesions (24). HAMSCs transplantation promotes ovarian function by inhibiting tumor necrosis factor-alpha-mediated cell apoptosis and reducing inflammation in chemotherapy-induced premature ovarian failure (25). HAMSCs are able to produce/release a number of biologically active cytokines/growth modulators, such as basic fibroblast growth factor (bFGF), epithelial growth factor (EGF), insulin growth factor-1 (IGF-1), stem cell factor (SCF), IL-1a, IL-10, insulin, tumor necrosis factor-a, IFN-g, and leukemia inhibitory factor (LIF), some of which could constitute crucial components in maintaining/enhancing the survival/anti-senescence/apoptosis of progenitor/adult cells (26). HAMSCs can secrete several cytokines and growth factors, promoting the survival of the surrounding cells. The cytokines and the growth factors, such as IL-6, M-CSF, IL-10, HGF, TGF- $\beta$ and PGE2 contribute to preventing apoptosis of injured pancreatic $\beta$-cells and enhancing regeneration of endogenous progenitor cells via angiogenic, cytoprotective, anti-inflammatory, mitogenic and anti-apoptotic effects (21).

It was reported that mitochondria use oxidative phosphorylation to convert dietary intake into ATP; in the process, they generate ROS, which can damage mitochondrial DNA, impair respiratory chain function, and cause nuclear DNA damage and cellular checkpoint activation (27). p53 is a transcription factor that plays a key role in both cell cycle arrest and apoptosis. p53 has many anticancer mechanisms and plays a role in apoptosis, genetic stability, and inhibition of senescence/apoptosis (28).
Present study was to investigate the potential molecular signaling pathways of UVA-induced HDF senescence engaged by HAMSCs. We found that HAMSCs promoted proliferation in UVA induced HDFs which confirmed by flow cytometry. SA- $\beta$-gel staining revealed that senescence of UVA irradiated HDFs co-cultured with HAMSCs decreased compared with HDFs accepted UVA irradiation only. ROS generation in UVA induced HDFs was determined to measure the anti-oxidant properties. The excessive production of ROS, such as superoxides and $\mathrm{H}_{2} \mathrm{O}_{2}$ severely damages the DNA, protein and lipids. Our findings suggest that HAMSCs inhibited ROS generation in UVA-induced HDFs. Expression of MMP, p53, p38, SIRT1 were also significantly increased in the co-culture group. By improving senescence against oxidative stress, HAMSCs might represent an appropriate therapeutic alternative against UVA induced skin aging.

Signal pathways involved in oxidative stress-induced inhibition of senescence consist of MAPK, Akt/mTOR/4EBP1, p53 and NFkB [Sreedhar et al (29), 2016]. The present study highlights the antioxidant role of HAMSCs in promoting UVA-induced proliferation and senescence. We found that activation of the ERK/MAPK signaling pathway is essential for protective effect against oxidative stress induced cell injury in HDFs. These data shed light on the molecular mechanism the signaling cascade mediated by HAMSCs and identify the potential role of HAMSCs in tissue engineering.

\section{Acknowledgements}

The present study was support by the Nanjing Medical Science and Technology Development Fund Project (grant no. 2015NJMUZD088).

\section{References}

1. He YY, Council SE, Feng L and Chignell CF: UVA-induced cell cycle progression is mediated by a disintegrin and metalloprotease/epidermal growth factor receptor/AKT/Cyclin D1 pathways in keratinocytes. Cancer Res 68: 3752-3758, 2008. 
2. Morita A, Grewe M, Grether-Beck S, Olaizola-Horn S and Krutmann J: Induction of proinflammatory cytokines in human epidermoid carcinoma cells by in vitro ultraviolet A1 irradiation. Photochem Photobiol 65: 630-635, 1997.

3. Min W, Liu X, Qian Q, Lin B, Wu D, Wang M, Ahmad I, Yusuf N and Luo D: The Effects of baicalin against UVA-induced photoaging in skin fibroblasts. Am J Chin Med 42: 709-727, 2014.

4. Ilancheran S, Michalska A, Peh G, Wallace EM, Pera M and Manuelpillai U: Stem cells derived from human fetal membranes display multilineage differentiation potential. Biol Reprod 77: 577-588, 2007.

5. Miki T, Lehmann T, Cai H, Stolz DB and Strom SC: Stem cell characteristics of amniotic epithelial cells. Stem Cells 23: $1549-1559,2005$

6. Song YS, Joo HW, Park IH, Shen GY, Lee Y, Shin JH, Kim H Shin IS and Kim KS: Transplanted human amniotic epithelial cells secrete paracrine proangiogenic cytokines in rat model of myocardial infarction. Cell Transplant 24: 2055-2064, 2015.

7. Chen YT, Li W, Hayashida Y, He H, Chen SY, Tseng DY, Kheirkhah A and Tseng SC: Human amniotic epithelial cells as novel feeder layers for promoting ex vivo expansion of limbal epithelial progenitor cells. Stem Cells 25: 1995-2005, 2007.

8. Lai D, Wang Y, Sun J, Chen Y, Li T, Wu Y, Guo L and Wei C: Derivation and characterization of human embryonic stem cells on human amnion epithelial cells. Sci Rep 5: 10014, 2015.

9. Banas R, Miller C, Guzik L and Zeevi A: Amnion-derived multipotent progenitor cells inhibit blood monocyte differentiation into mature dendritic cells. Cell Transplant 23: 1111-1125, 2014.

10. Díaz-Prado S, Muiños-López E, Hermida-Gómez T, Cicione C, Rendal-Vázquez ME, Fuentes-Boquete I, de Toro FJ and Blanco FJ: Human amniotic membrane as an alternative source of stem cells for regenerative medicine. Differentiation 81: 162-171, 2011.

11. Díaz-Prado S, Muiños-López E, Hermida-Gómez T, Rendal-Vázquez ME, Fuentes-Boquete I, de Toro FJ and Blanco FJ: Multilineage differentiation potential of cells isolated from the human amniotic membrane. J Cell Biochem 111: 846-857, 2010

12. Han K, Lee JE, Kwon SJ, Park SY, Shim SH, Kim H, Moon JH, Suh CS and Lim HJ: Human amnion-derived mesenchymal stem cells are a potential source for uterine stem cell therapy. Cell Prolif 41: 705-725, 2008.

13. Onishi R, Onishi S, Higashi R, Yamahara K, Yoshimatsu J, Katsurada T, Okubo N, Nakagawa K, Takeda H and Sakamoto N: The anti-inflammatory effect of human annion-derived mesenchymal stem cells. Placenta 35: A23, 2014.

14. Lee JH, Ryu IH, Kim EK, Lee JE, Hong S and Lee HK: Induced expression of insulin-like growth factor-1 by amniotic membrane-conditioned medium in cultured human corneal epithelial cells. Invest Ophthalmol Vis Sci 47: 864-872, 2006

15. Ohno-Matsui K, Ichinose S, Nakahama K, Yoshida T, Kojima A, Mochizuki $\mathrm{M}$ and Morita I: The effects of amniotic membrane on retinal pigment epithelial cell differentiation. Mol Vis 11: $1-10,2005$

16. Akrami H, Soheili ZS, Sadeghizadeh M, Khalooghi K, Ahmadieh H, Kanavi MR, Samiei S and Pakravesh J: Evaluation of RPE65, CRALBP, VEGF, CD68, and tyrosinase gene expression in human retinal pigment epithelial cells cultured on amniotic membrane. Biochem Genet 49: 313-322, 2011.
17. Yan ZJ, Zhang P, Hu YQ, Zhang HT, Hong SQ, Zhou HL, Zhang MY and Xu RX: Neural stem like cells derived from human amnion tissue are effective in treating traumatic brain injury in rat. Nerochem Res 38: 1022-1033, 2013.

18. Nogami M, Tsuno H, Koike C, Okabe M, Yoshida T, Seki S, Matsui Y, Kimura T and Nikaido T: Isolation and characterization of human amniotic mesenchymal stem cells and their chondrogenic differentiation. Transplantation 93: 1221-1228, 2012.

19. Lim H, Han K, Lee J, Shim S, Moon J, Suh C, Kim J and Kim H: Human amnion derived mesenchymal stem cells may have potential to contribute and differentiate endometrial cells in vivo. Hum Reprod 22: i171, 2007.

20. Tamagawa T, Ishiwata I, Ishikawa H and Nakamura Y: IInduced in-vitro differentiation of neural-like cells from human amnion-derived fibroblast-like cells. Hum Cell 21: 38-45, 2008.

21. Niknejad H, Yazdanpanah G and Ahmadiani A: Induction of apoptosis, stimulation of cell-cycle arrest and inhibition of angionenesis make human amnion-derived cells promising sources for cell therapy of cancer. Cell Tissue Res 363: 599-608, 2016.

22. Lei LT, Chen JB, Zhao YL, Yang SP and He L: Resveratrol attenuates senescence of adipose-derived mesenchymal stem cells and restores their paracrine effects on promoting insulin secretion of INS-1 cells through Pim-1. Eur Rev Med Pharmacol Sci 20: 1203-1213, 2016.

23. Kawakubo K, Ohnishi S, Fujita H, Kuwatani M, Onishi R, Masamune A, Takeda $\mathrm{H}$ and Sakamoto N: Effect of fetal membrane-derived mesenchymal stem cell transplantation rats with acute and chronic pancreatitis. Pancreas 45: 707-713, 2016.

24. Ono M, Ohnishi S, Honda M, Ishikawa M, Hosono H, Onishi R, Nakagawa K, Takeda H and Sakamoto N: Effects of human amnion-derived mesenchymal stromal cell transplantation in rats with radiation proctitis. Cytotherapy 17: 1545-1559, 2015.

25. Li J, Koike-Soko C, Sugimoto J, Yoshida T, Okabe M and Nikaido T: Human amnion-derived stem cells have immunosuppressive properties on NK cells and monocytes. Cell Transplant 24: 2065-2076, 2015.

26. Sahin E, Colla S, Liesa M, Moslehi J, Müller FL, Guo M, Cooper M, Kotton D, Fabian AJ, Walkey C, et al: Telomere dysfunction induces metabolic and mitochondrial compromise. Nature 470: 359-365, 2011.

27. Hengartner MO: The biochemistry of apoptosis. Nature 407: 770-776, 2000.

28. Vogelstein B, Lane D and Levine AJ: Surfing the p53 network. Nature 408: 307-310, 2000

29. Sreedhar R, Giridharan W, Arumugam S, Karuppagounder V, Palaniyandi SS, Krishnamurthy P, Quevedo J, Watanabe K, Konishi T and Thandavarayan RA: Role of MAPK-mediated endoplasmic reticulum stress signaling in the heart during aging in senescence-accelerated prone mice. Biofactors 42: 368-375, 2016. 